\title{
The detection of pulsed emission at the spin-period of the white dwarf in AE Aquarii in MeerKAT and Fermi-LAT data.
}

\section{S.T. Madzime, ${ }^{a, *}$ P.J. Meintjes, ${ }^{a}$ H.J. van Heerden, ${ }^{a}$ K.K. Singh, ${ }^{a, b}$ D.A.H. Buckley, ${ }^{a, c}$}

\section{P.A. Woudt ${ }^{d}$ and R. Fender ${ }^{e}$}

${ }^{a}$ Department of Physics, University of the Free State, Bloemfontein, 9301, South Africa

${ }^{b}$ Astrophysical Sciences Division, Bhabha Atomic Research Centre, Mumbai 400085, India

${ }^{c}$ South African Astronomical Observatory (SAAO), Observatory, 7925, Cape Town, South

${ }^{d}$ Department of Astronomy, University of Cape Town (UCT), Rondebosh, Cape Town,7700 South Africa

${ }^{e}$ Department of Physics, University of Oxford, Oxford, OX1 3PU, United Kingdom

E-mail: tsmadzime@gmail.com, MeintjPJ@ufs.ac.za, VanHeerden@ufs.ac.za, kksastro@barc.gov.in, dibnob@saao.ac.za, WoudtPA@ast.uct.ac.za, Rob.Fender@physics.ox.ac.uk

Here we present the first report of pulsed emission at the spin period of the white dwarf in MeerKAT data, which is also the first report of pulsed emission at the spin period of the white dwarf in radio frequencies (L-Band). Further support for the pulsar-like behaviour of AE Aquarii is the detection of pulsed emission at the spin period of the white dwarf in AE Aquarii in Fermi-LAT data. By isolating data sections that show emission above the $2 \sigma$ level our periodicity analysis reveal clear indications of pulsed emission at the spin period of the white dwarf and its associated second harmonic (first overtone), which implies particle acceleration from both poles of the white dwarf. The detection of pulsed emission in Fermi-LAT data mimic to a large extent the emission profiles detected in very high energy gamma-ray observations made by two independent groups at Pothefstroom and Durham in the late 1980's to early 1990's. Furthermore, Fermi-LAT data sections that were observed simultaneously with optical flares show clear pulsed emission patterns at the spin period of the white dwarf similar to those reported in the late 1980's. The results presented here confirm AE Aquarii as a transient gamma-ray source with clear indications of spectral hardening during periods with enhanced gamma-ray activity. It is also shown that $\mathrm{AE}$ Aquarii may be detectable with CTA. A strategy is presented on how detectable pulsed emission may be extracted from the data.

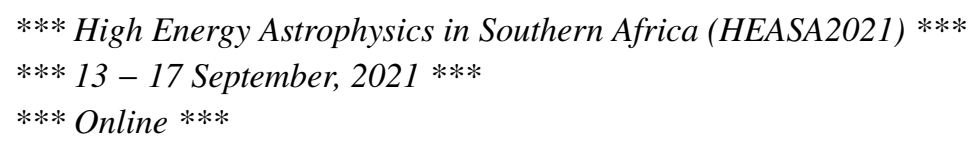

\footnotetext{
${ }^{*}$ Speaker
} 


\section{Introduction}

The novalike cataclysmic variable AE Aquarii (AE Aqr) is a close binary system, which

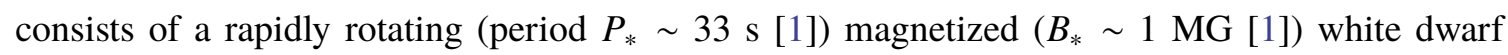
(WD), and a late-type main sequence companion (K4-5) star. The WD in AE Aqr is orbiting the secondary star with an orbital period of $9.88 \mathrm{hrs}$ (e.g., [2-4]). Optical wavelengths in AE Aqr have visual magnitude varying between $m_{V}=10$ and $m_{V}=12$ [5]. This system exhibits highly transient multiwavelength emission with their characteristics revealed by several studies: optical (e.g., [1, 4, 6]), radio (e.g., [7-9]), X-rays (e.g., [10, 11]) and possibly in $\mathrm{TeV}$ gamma-rays energy bands (e.g., [12-14]). It has been shown ([8]) that the variability in the observed radio emission corresponds to the integrated emission form several expanding synchrotron emitting clouds in different phases of their evolution, which presents a rather flat spectrum with time averaged flux

$$
\langle S(v, \rho)\rangle \propto v^{5 / 2(2-\epsilon)}
$$

where $\rho$ is a dimensionless radius of an expanding blob and $\epsilon$ is the index of the flare occurance spectrum (see [8] for a detailed discussion), which is assumed to be (e.g. [8])

$$
f(S(v, \rho)) \propto S(\nu, \rho)^{-\epsilon},
$$

meaning that low-energy flares occur more regularly than high-energy flares. It has been shown ([8]) that the observed time-average spectrum corresponds to $\epsilon \sim 2.2$, resulting in,

$$
\langle S(v, \rho)\rangle \propto v^{0.5} .
$$

The first reports of Very High Energy (VHE) gamma-ray emission from the enigmatic AE Aqr were reported by two independent groups (e.g. [12, 13, 15]) in the late 1980's to early 1990's. The gamma-ray emission from AE Aqr were described as sporadic, aperiodic and burst-like with pulsations at or close to the WD's spin period. On occasions the pulsations were also seen at the second harmonic (16.54 s) of the spin period. The follow-up studies using other Cherenkov telescopes resulted in no detection of any gamma-ray emission (e.g. [16]). However, the discovery of hard X-rays $[17,18]$ with a spectrum that resembles a power-law, rekindled the notion that WDs are potential particle accelerators and hence possible gamma-ray sources. A follow-up study for pulsed gamma-ray emission using the Fermi Large Area Telescope (Fermi-LAT) pass 7 and pass 8 data resulted in low-level but consistent pulsed emission at or close to the spin period (33.08 s) and the second harmonic (16.54 s) of the WD in AE Aqr [19-21]. These results combined with new Fermi-LAT pass 8 data and the South African interferometer array telescope MeerKAT, motivated a new follow-up gamma-ray and radio study of AE Aqr. The paper is structured as follows: The observations, analysis and results of the radio emission from AE Aqr will be reported in the first section, followed by the analysis and results of AE Aqr's Fermi-LAT data, and finally some conclusions.

\section{MeerKAT Observations and Results of AE Aqr}

AE Aqr was observed on 25 February 2019 by the MeerKAT telescope array, the precursor of the square kilometre array (SKA). This observation was performed under a ThunderKAT project 
[22]. This source was observed for a total of 45 minutes in three identical scans with PKS B1934-638 as the bandpass and flux calibrator source which was observed for 5 mins at the onset of the observation. The amplitude and phase calibrator source PKS 2008-068, observed for 2 mins after each scan, can be seen as gaps in the light curves. The light curves of this observation presented in Figure 1 are; lower L-Band (856-1140 MHz), middle L-Band (1140-1426 MHz) and upper L-Band (1426-1712 MHz), and finally the total L-Band (856-1712 MHz). Radio emission from AE Aqr displays high levels of variability in the light curves. This variability is most probably the result of the interaction between the magnetosphere of the WD and the propeller ejected matter since the emission from AE Aqr is not driven by the direct accretion of mass onto the surface of the WD.

The search for spin modulation of AE Aqr's radio emission in the MeerKAT data was conducted using the Lomb-Scargle [23, 24] technique available in AstroPy. In Figure 1, we present the first detection of pulsed radio emission from AE Aqr. To supplement the power spectrum of the main L-Band (see Figure 1 (a)) over a wide frequency range the power spectra of the lower, middle and upper L-Band were added incoherently to amplify the periodic signal (see Figure 1 (b)). The technique of stacking is useful to distinguish between erroneous detection and signal. If $n$ observations are conducted at a given frequency $v$, the chance probabilities may be integrated incoherently to get a more significant pulsed signal at the cost of phase information. The technique sums up all power with same frequencies, to obtain a statistic which follows $\chi^{2}$ distribution with $2 n$ degrees of freedom [25]. The L-Band spectrum of AE Aqr shows a power-law (see Figure 2 ), which may associate AE Aqr's radio emission with non-thermal synchrotron emission. The mechanism behind the measured pulsed signal is currently under investigation. However, the radio flux measurements displays a non-thermal origin which can be modelled with a power-law spectrum in the L-Band (see Figure 2). The total L-Band average flux density is also included in the SED on the right, which shows that the observed average flux density in the L-Band correlates with the overall time-averaged flux density profile SED from radio to infra-red frequencies [26] which is the typical signature of the non-thermal synchrotron emission of a superposition of expanding synchrotron emitting clouds $([8,27])$.

\section{Fermi-LAT gamma-ray data analysis and results}

The search for steady gamma-ray emission was conducted using binned and unbinned standard analysis utilizing the upgraded Fermi-LAT pass 8 dataset. The periodic modulation of gamma-ray emission was conducted using gtpsearch and gtpphase routines of Fermi Science Tools software packages. The search for steady gamma-ray emission using the $\sim 11$-year pass 8 dataset and utilizing standard analysis techniques revealed no gamma-ray emission above the background.

A 14-day light curve produced from an 11-year dataset revealed sections in the light curve with gamma-ray emission above the $2 \sigma$ significance level. Some of these sections display pulsed emission near the WD's spin period [21]. Investigating 10 minute dataset intervals, and searching for periodic modulation, revealed more significant pulses at or close to the spin period of the WD and its second harmonic. The power spectra of these sections with significance of $\geq 3 \sigma$ at the same frequency were incoherently combined (stacked). The stacked power spectra display pulsations at $33.08 \mathrm{~s}$ and $16.54 \mathrm{~s}$ (see Figure 3). In Figure 3 off-centre stacked power spectra are displayed, 

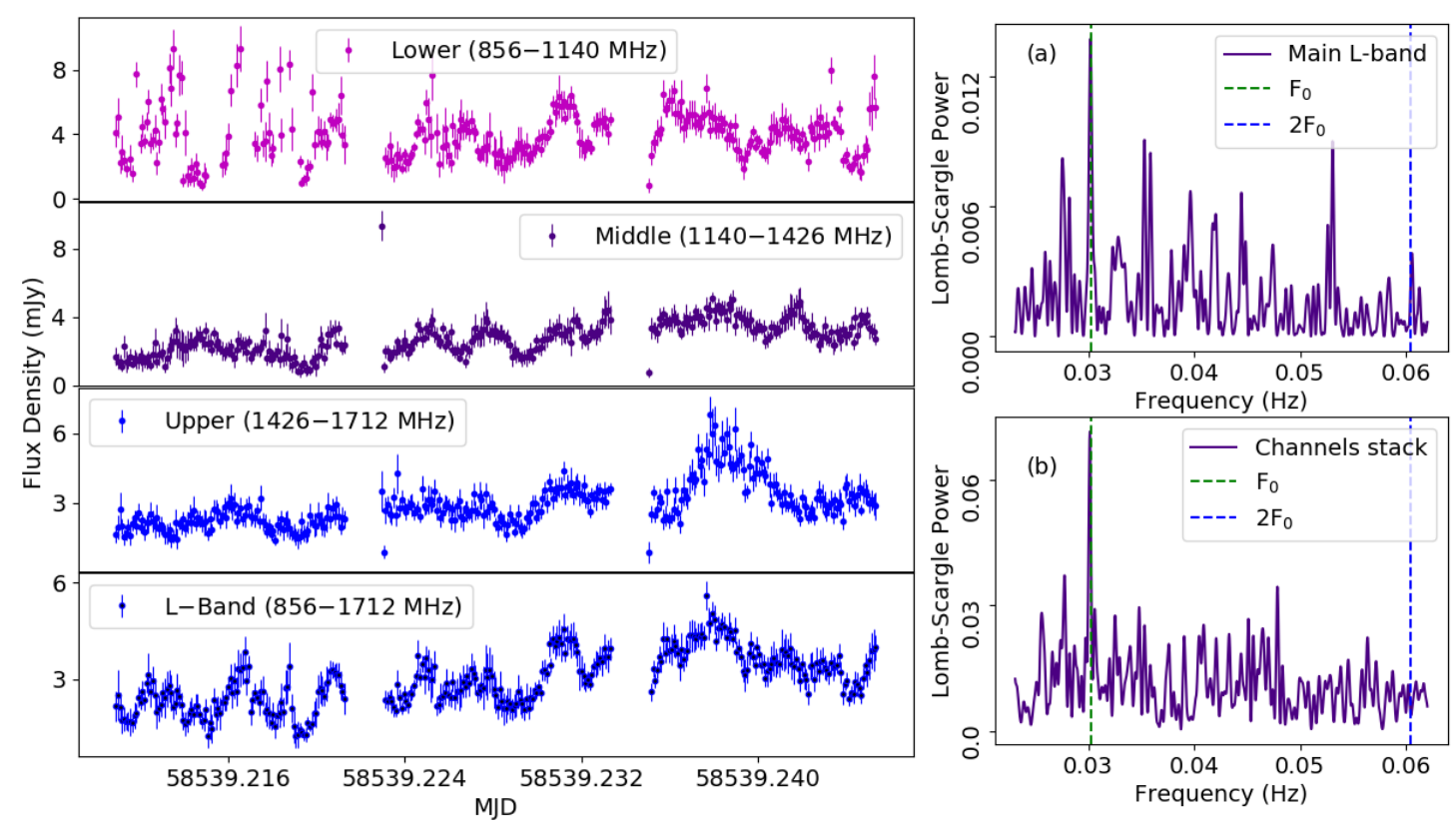

Figure 1: (Left panel) The L-Band data was binned in 8 second intervals and divided into channels from top: lower (856-1140 MHz), middle (1140-1426 MHz) and upper (1426-1712 MHz) channel light curves, and the bottom light curve represent the total L-Band (856-1712 MHz) light curve. (a) The power spectrum of AE Aqr produced from the MeerKAT L-Band observation on 25 February 2019. There is a strong indication of pulsed emission at the WD's spin period $\left(\mathrm{F}_{0}=30.23 \mathrm{mHz}\right)$. (b) This represents the incoherently combined (stacked) power spectrum produced from lower, middle and upper channel light curves.
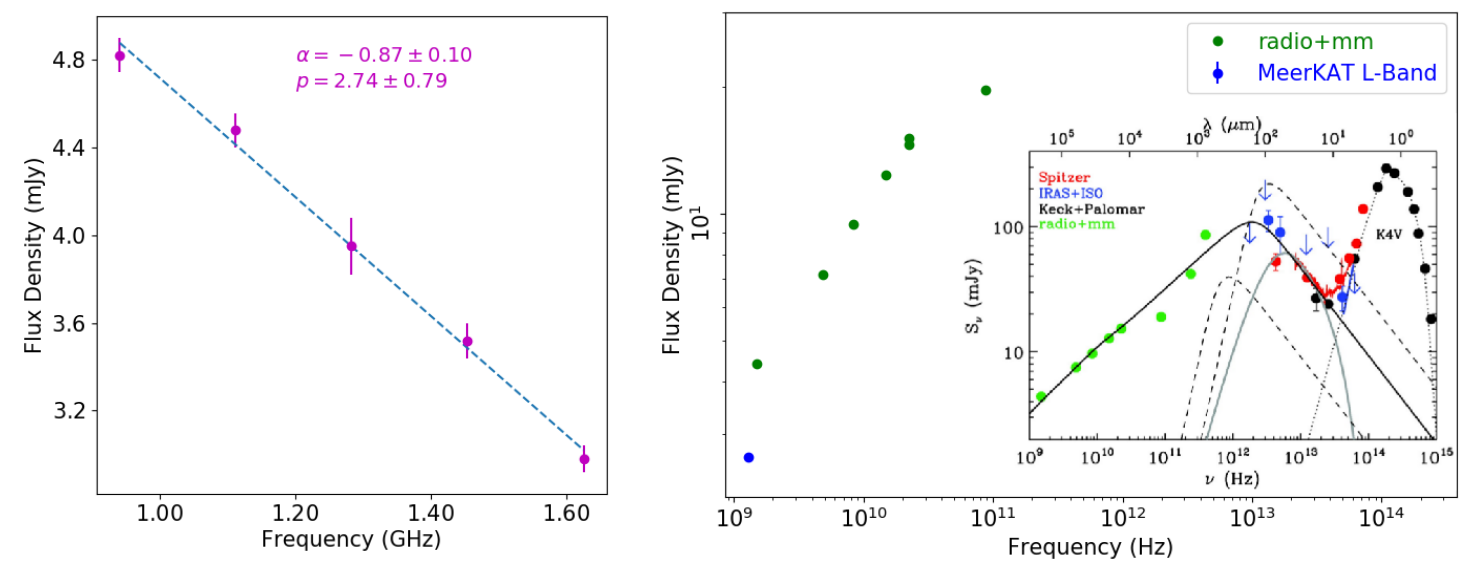

Figure 2: The left panel shows MeerKAT's spectral points of AE Aqr extracted from the L-Band and the right panel is the time-average spectral energy distribution (SED) of AE Aqr over a wide frequency range. The positive slope at lower frequencies is compatible to the integrated emission of a superposition of synchrotron flares from expanding synchrotron emitting clouds that turn from optically thin to optically thick at lower frequencies, i.e. the so-called van der Laan process (e.g. $[8,27])$. The avearge L-Band flux density in the entire MeerKAT observation is indicated by the blue solid circle and the rest of the data as well as the insert was obtained from Dubus et al,. [26] for comparison. 

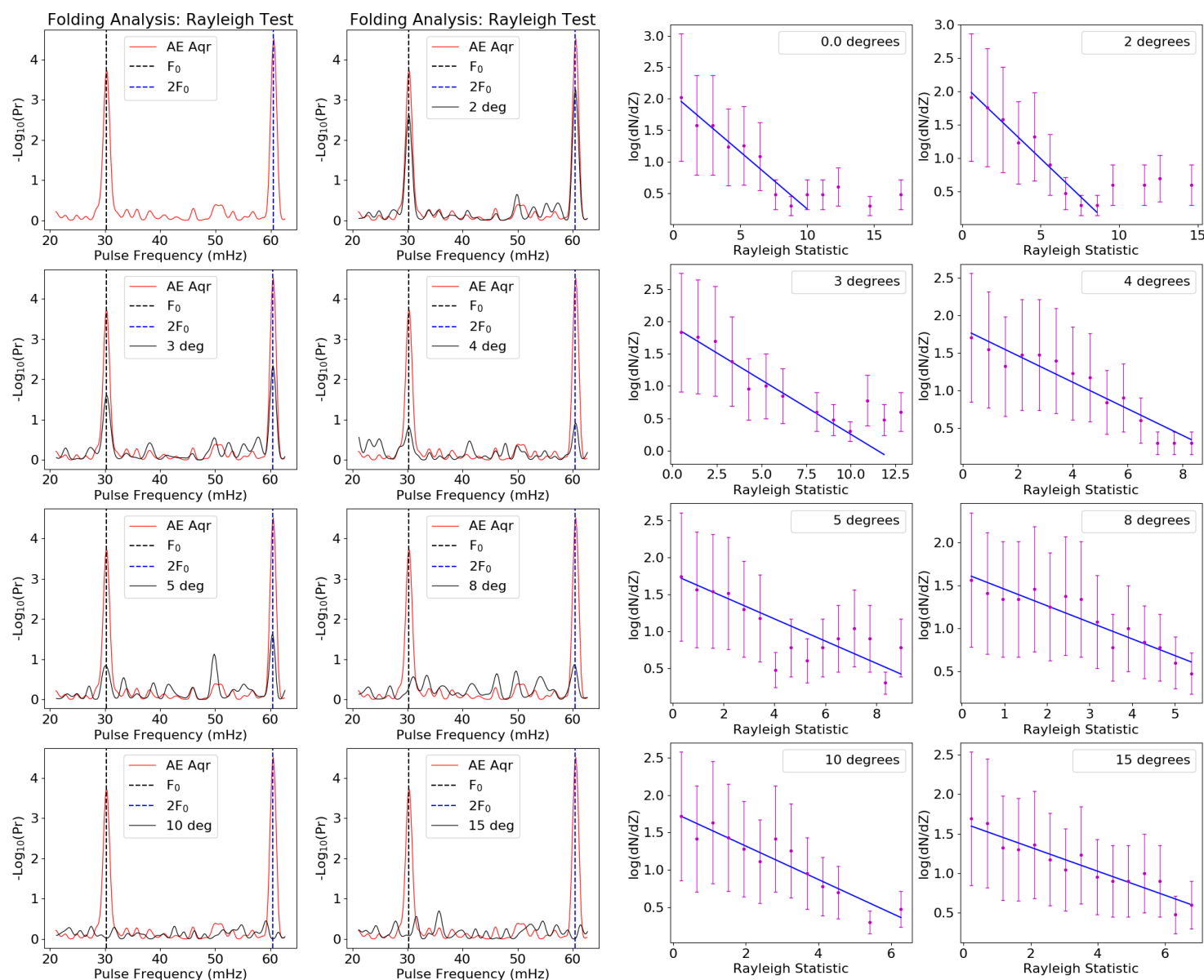

Figure 3: First two columns represents Fermi-LAT stacked power spectra and the last two columns are noise profile centred at the position of AE Aqr ( 0.0 degrees) and control analysis demonstrating how the power in the power spectrum return to white noise further away from AE Aqr, which illustrates that the pulsed power is confined to AE Aqr's position in the sky.

which show that the spin modulation power gradually decreases away from the source. These power spectra were produced to test whether these pulsations are not spurious detections. We produce the power spectra by using positions in the sky consecutively further away from $\mathrm{AE}$ Aqr $\left(3^{\circ}-15^{\circ}\right)$. The on-source noise profile at the $33.08 \mathrm{~s}$ and $16.54 \mathrm{~s}$ periods deviates from the theoretical white noise distribution for the power spectra produced for data corresponding to AE Aqr's position in the sky. The power conforms to a white noise distribution for off-centre power spectra $\left(3^{\circ}-15^{\circ}\right)$. To search for gamma-ray pulses that coincides with Fermi-LAT pulses, sections that correspond to optical flares observed with the University of the Free State (UFS-Boyden) $1.5 \mathrm{~m}$ telescope (see Figure 4) were used for periodic analysis. Indications of periodic gamma-ray emission were identified from the power spectra that correspond to the optical flares, and these power spectra were incoherently stacked (see Figure 4). The stacked spectra that coincides with enhanced optical activity exhibit pulsed emission close to the WD's spin period. These result corresponds well with previously reported pulsed behaviour of VHE emission during optical flares [13, 28]. However, the weak signal which was present in the vicinity of second harmonic of each spectra disappear after 
stacking.

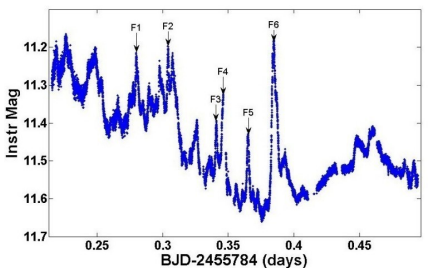

(a)

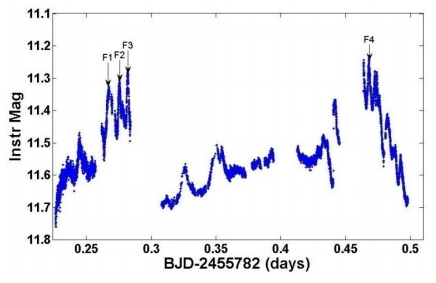

(c)

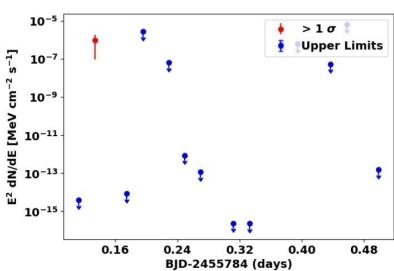

(b)

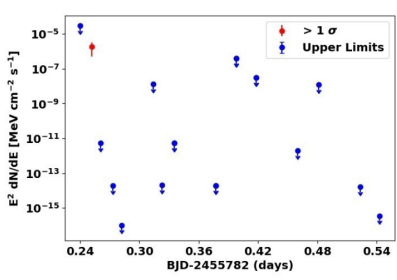

(d)

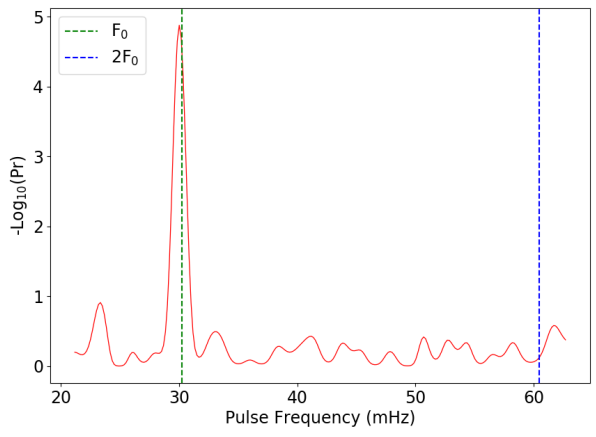

Figure 4: The first column: (a) and (c) are lightcurves of optical photometry conducted using the UFS-Boyden $1.5 \mathrm{~m}$ telescope near Bloemfontein (South Africa). Second column: (b) and (d) gamma-ray data that coincided with optical flares. The last column: power spectrum of combined spectra produced from the Fermi-LAT data sections that coincide with optical flares.
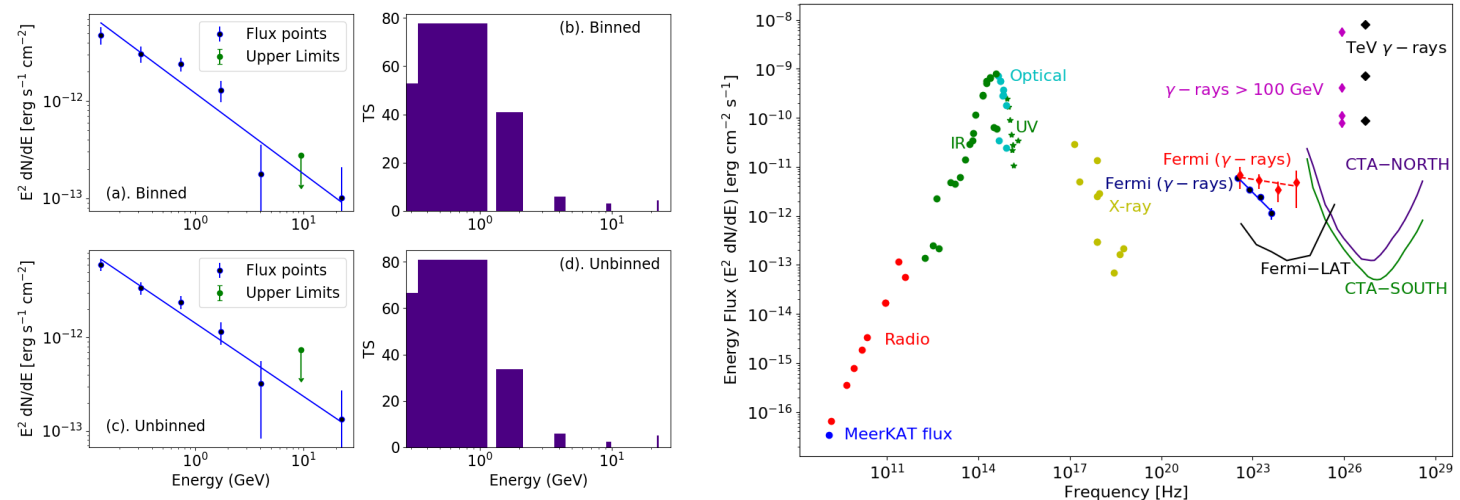

Figure 5: The spectral points are fitted with a power-law with a photon index $\Gamma \sim 2$ (with goodness of the fit $\chi^{2} \sim 12$ ( $p$-value $\left.\sim 0.025\right)$ with $n=6$ degrees of freedom). The last panel display the broadband SED of AE Aqr from radio to VHE emission (see e.g., [18]) including CTA, and Fermi-LAT sensitivity curves. The Fermi-LAT gamma-ray spectra for quiescence (navy blue solid circles) and flare sections (red diamonds) of the data indicate a spectral hardening.

The binned and unbinned analysis associated with data sections with $>0 \sigma$ significance levels resulted in an average significance of $>14 \sigma$. The gamma-ray spectra for both display a power-law model with photon index $\Gamma \sim 2$ (see Figure 5 (a) and (c)). Notable from the significance energy distribution Figure 5 (b) and (d), is the significance of gamma-ray emission, which seems to be high in the lower energy bins. The spectra associated with data sections with significance above $2 \sigma$ can be described by a power-law, where spectral hardening is also observed (see Figure 5, last column) which may result in detectable flux levels during periods of enhanced activity. These 
results may put AE Aqr in the spotlight for follow-up studies with more sensitive Air Cherenkov detectors like CTA.

\section{Conclusion}

We have detected non-thermal pulsed radio emission from AE Aqr in the L-Band MeerKAT data. This marks the first time pulsed radio emission is reported from this source. The measured spectral energy can be fitted with a power-law profile. Fermi-LAT data sections that display transient events reveal AE Aqr in both binned and unbinned analyses, with an average significance of $\sim 14 \sigma$ for the selective filtered data. This increase in the significance of the gamma-ray signal using a more selective filtered data validates the search for periodic emission in data sections that showed enhanced levels of gamma-ray activity $(\geq 2 \sigma)$. Periodogram analysis and stacking of power spectra revealed a clear indication of pulsed emission at the spin frequency and some occurrences was also seen on the second harmonic. However, the second harmonic was not present in the stacked power spectra of the data that coincides with the optical flares or the MeerKAT data. A control analysis revealed that the power at the fundamental frequency and the second harmonic fades away in region of the sky away from AE Aqr. It was also shown that the gamma-ray spectra of the flaring periods are constantly harder than the overall spectrum, which predicts flux levels above the CTA threshold.

\section{References}

[1] J. Patterson, Rapid oscillations in cataclysmic variables. III-an oblique rotator in an AE Aquarii, The Astrophysical Journal 234 (1979) 978.

[2] A. H. Joy, Spectroscopic Observations of AE Aquarii., The Astrophysical Journal 120 (1954) 377.

[3] J. A. Crawford and R. P. Kraft, An Intrepretation of AE Aquarii., The Astrophysical Journal 123 (1956) 44.

[4] G. Chincarini and M. Walker, Image tube spectroscopic studies of rapid variables. $I V$-Spectroscopic and photometric observations of AE Aquarii, Astronomy and Astrophysics 104 (1981) 24.

[5] E. Zinner, Mitteilungen übr veränderliche und verdächtige sterne, Astronomische Nachrichten 265 (1938) 345.

[6] M. Eracleous and K. Horne, The speedy magnetic propeller in the cataclysmic variable AE Aquarii, The Astrophysical Journal 471 (1996) 427.

[7] J. Bookbinder and D. Lamb, Discovery of radio emission from AE Aquarii, The Astrophysical Journal 323 (1987) L131.

[8] T. Bastian, G. Dulk and G. Chanmugam, Radio flares from AE Aquarii-A low-power analog to Cygnus X-3?, The Astrophysical Journal 324 (1988) 431. 
[9] M. Abada-Simon, A. Lecacheux, T. S. Bastian, J. A. Bookbinder and G. A. Dulk, The spectrum and variability of radio emission from AE Aquarii, The Astrophysical Journal 406 (1993) 692.

[10] J. Patterson and C. Price, Rapid oscillations in cataclysmic variables. v-h2252-035, a single-sideband X-ray and optical pulsar, The Astrophysical Journal 243 (1981) L83.

[11] Y. Terada, M. Ishida, K. Mukai, T. Dotani, K. Makishima, S. Naik et al., Possible Suzaku detection of non-thermal $X$-ray signals from a rotating magnetized white dwarf, Advances in Space Research 41 (2008) 512.

[12] P. J. Meintjes, Simultaneous optical and TeV gamma-ray observations of the cataclysmic variable AE Aquarii, Ph.D. thesis, 1992.

[13] P. Meintjes, O. De Jager, B. Raubenheimer, H. Nel, A. North, D. Buckley et al., Simultaneous optical and TeV gamma-ray observations of the cataclysmic variable AE Aquarii, The Astrophysical Journal 434 (1994) 292.

[14] P. Chadwick, J. Dickinson, M. Dickinson, N. Dipper, J. Holder, T. McComb et al., A burst of pulsed VHE gamma rays from AE Aquarii, Astroparticle Physics 4 (1995) 99.

[15] C. Bowden, S. Bradbury, P. Chadwick, J. Dickinson, N. Dipper, P. Edwards et al., $350 \mathrm{GeV}$ gamma rays from AE Aqr, Astroparticle Physics 1 (1992) 47.

[16] J. Aleksić, S. Ansoldi, L. A. Antonelli, P. Antoranz, A. Babic, P. Bangale et al., Magic search for vhe $\gamma$-ray emission from AE Aquarii in a multiwavelength context, Astronomy \& Astrophysics 568 (2014) A109.

[17] Y. Terada, T. Hayashi, M. Ishida, K. Mukai, T. Dotani, S. Okada et al., Suzaku discovery of hard X-ray pulsations from a rotating magnetized white dwarf, AE Aquarii, Publications of the Astronomical Society of Japan 60 (2008) 387.

[18] B. Oruru and P. Meintjes, X-ray characteristics and the spectral energy distribution of $A E$ Aquarii, Monthly Notices of the Royal Astronomical Society 421 (2012) 1557.

[19] H. J. van Heerden and P. J. Meintjes, Fermi-LAT analysis and upper-limit calculations for AE Aquarii., Mem. Soc. Astron. Ital. 86 (2015) 111.

[20] J. Li, D. F. Torres, N. Rea, E. de Ona Wilhelmi, A. Papitto, X. Hou et al., Search for gamma-ray emission from AE Aquarii with seven years of Fermi-LAT observations, The Astrophysical Journal 832 (2016) 35.

[21] S. T. Madzime, P. Meintjes, K. K. Singh and H. van Heerden, Search for gamma-ray emission from the nova-like variable AE Aquarii using the Fermi-LAT Pass 8 data Archive, in Proceedings of 7th Annual Conference on High Energy Astrophysics in Southern AfricaPoS(HEASA2019), vol. 371, p. 051, 2020, DOI. 
[22] P. A. Woudt, R. Fender, S. Corbel, M. Coriat, F. Daigne, H. Falcke et al., ThunderKAT: The MeerKAT Large Survey Project for Image-Plane Radio Transients, in Proceedings of MeerKAT Science: On the Pathway to the SKA - PoS(MeerKAT2016), vol. 277, p. 013, 2018, DOI.

[23] N. R. Lomb, Least-squares frequency analysis of unequally spaced data, Astrophysics and space science 39 (1976) 447.

[24] J. D. Scargle, Studies in astronomical time series analysis. ii-statistical aspects of spectral analysis of unevenly spaced data, The Astrophysical Journal 263 (1982) 835.

[25] W. T. Eadie, D. Drijard and F. E. James, Statistical methods in experimental physics. Amsterdam: North-Holland, 1971.

[26] G. Dubus, R. E. Taam, C. Hull, D. M. Watson and J. C. Mauerhan, Spitzer space telescope observations of the magnetic cataclysmic variable AE Aquarii, The Astrophysical Journal 663 (2007) 516.

[27] P. Meintjes and L. Venter, Modelling the continuous radio outbursts in AE Aquarii, Monthly Notices of the Royal Astronomical Society 341 (2003) 891.

[28] P. Meintjes, B. Raubenheimer, O. De Jager, C. Brink, H. Nel, A. North et al., AE Aquarii an emitter of pulsed TeV gamma rays resembling optical emission during flares, The Astrophysical Journal 401 (1992) 325. 